\title{
Salmonella Urinary Tract Infection and Bacteremia Following Non-Typhoidal Salmonella Gastroenteritis: An Unusual Presentation
}

\author{
Areeba Altaf ${ }^{1}$, Numra Tunio ${ }^{2}$, Sameer Tunio ${ }^{3}$, Meer R. Zafar ${ }^{4,5}$, Numra Bajwa ${ }^{1}$ \\ 1. Internal Medicine, University at Buffalo/Catholic Health System, Buffalo, USA 2. Internal Medicine, Ziauddin \\ University, Karachi, PAK 3. Internal Medicine, Aga Khan University Hospital, Karachi, PAK 4. Internal Medicine, Sisters \\ of Charity Hospital, Buffalo, USA 5. Internal Medicine, Jacobs School of Medicine and Biomedical Sciences, Buffalo, \\ USA
}

Corresponding author: Areeba Altaf, areebaal@buffalo.edu

\begin{abstract}
Salmonella is primarily known to affect the gastrointestinal tract but can rarely cause infections at uncommon sites, such as the urinary tract. It is known that Salmonella can infect the urinary tract directly by blood, fecal contamination of urethra, urolithiasis, or secondary intraluminal ascending infection.

Our patient is a 59-year-old female with a past medical history of nephrolithiasis and multiple urinary tract infections (UTI) who presented with altered mental status and sepsis complicated by Salmonella bacteremia and UTI. Urine and blood cultures revealed Salmonella species > 100,000 colony-forming units per milliliter $(\mathrm{CFU} / \mathrm{mL})$ and non-typhoidal Salmonella, respectively. During the course of her hospital admission, the patient was treated with multiple antibiotics.
\end{abstract}

On further review, it was noted that the patient had presented to the emergency room (ER) about four months earlier with abdominal pain and watery diarrhea with a stool culture being positive for nontyphoidal Salmonella.

Gastroenteritis, sepsis, and enteric fever are normally known with Salmonella enterica serotype Typhi (S. Typhi). Less common extraintestinal diseases like UTI are due to non-typhoidal Salmonella. The most frequent pathogenesis of Salmonella UTI is probably hematogenous. UTI caused by non-typhoidal Salmonella is usually associated with structural abnormalities of the urinary tract. In our case, the patient had non-typhoidal Salmonella gastroenteritis followed by non-typhoidal Salmonella bacteremia and UTI.

Review began 10/21/2020 Review ended 12/06/2020 Published 12/20/2020

\section{() Copyright 2020}

Altaf et al. This is an open access article distributed under the terms of the Creative Commons Attribution License CC-BY 4.0., which permits unrestricted use, distribution, and reproduction in any medium, provided the original author and source are credited.
Categories: Internal Medicine, Urology, Infectious Disease

Keywords: non-typhoidal salmonella (nts), salmonella infection, gastroenteritis, urinary tract infection, bacteremia

\section{Introduction}

Salmonella is primarily known to affect the gastrointestinal tract but can rarely cause infections at uncommon sites like the urinary tract [1]. Non-typhoidal Salmonella (NTS) urinary tract infections were found to represent only $0.63 \%$ of all Salmonella infections in a large retrospective review [2]. It is known that Salmonella can infect the urinary tract directly by blood, fecal contamination of urethra, urolithiasis, or secondary intraluminal ascending infection.

Structural abnormality of the urinary tract, immunodeficiency, and chronic illness are predisposing factors for NTS urinary tract infections [3-4]. We present a case of altered mental status, nephrolithiasis, and sepsis complicated by Salmonella bacteremia and Salmonella urinary tract infection.

\section{Case Presentation}

A 59-year-old female with a past medical history of fibromyalgia, chronic obstructive pulmonary disease (COPD), allergic rhinitis, nephrolithiasis, and multiple urinary tract infections presented with altered mental status. On examination, she was febrile, tachycardic, and lethargic but arousable. No other significant examination findings, besides left flank pain and suprapubic tenderness, were noted. Her laboratory studies revealed abnormal liver enzymes, elevated creatinine and lactic acid, hypokalemia, high anion gap, and leukocytosis. Urinalysis was significant for infection.

Computed tomography (CT) of the abdomen/pelvis revealed acute obstructing calculi in the distal left ureter, left renal edema, and hydroureteronephrosis (Figures 1-2). 


\section{Cureus}

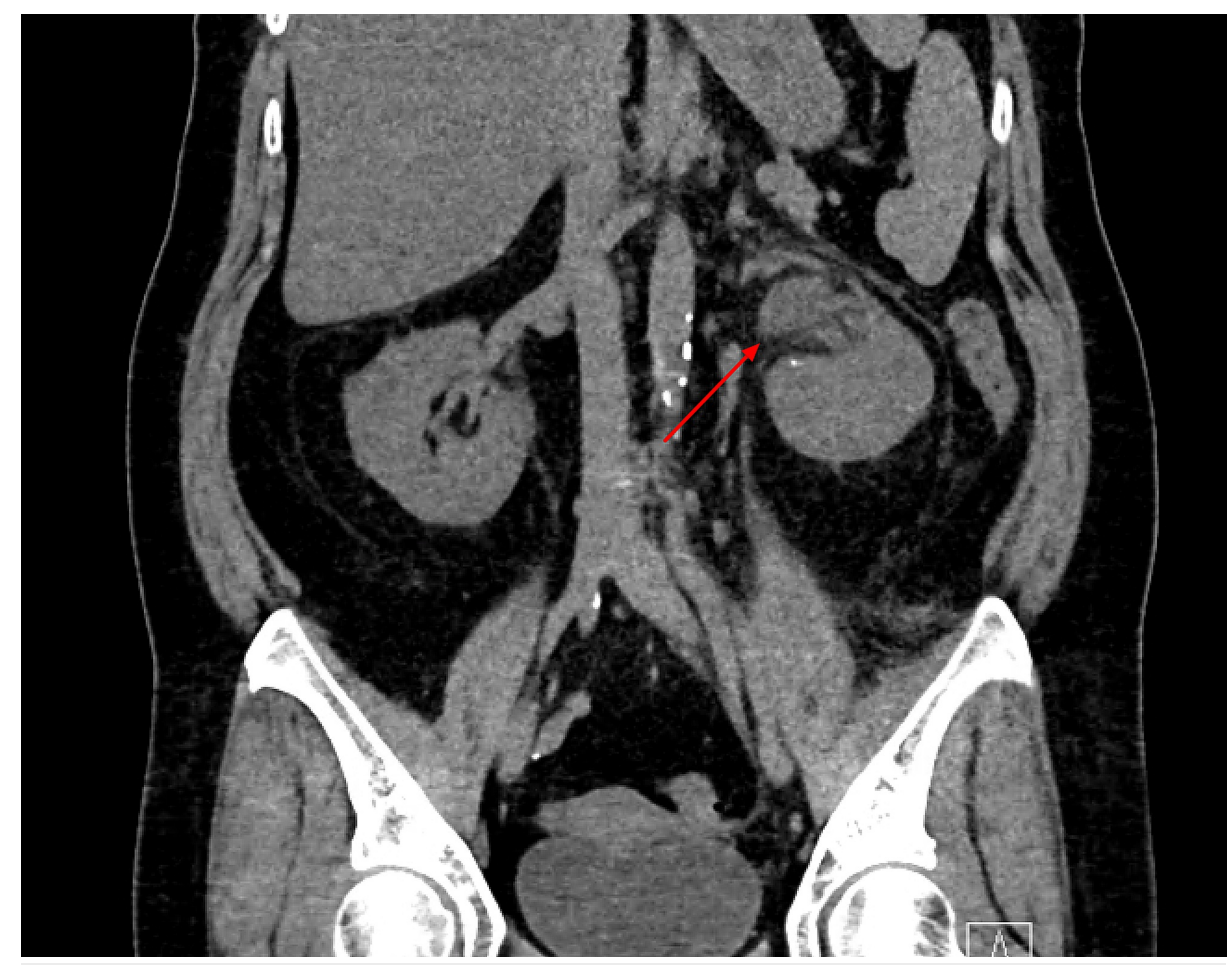

FIGURE 1: Left renal edema and hydroureteronephrosis

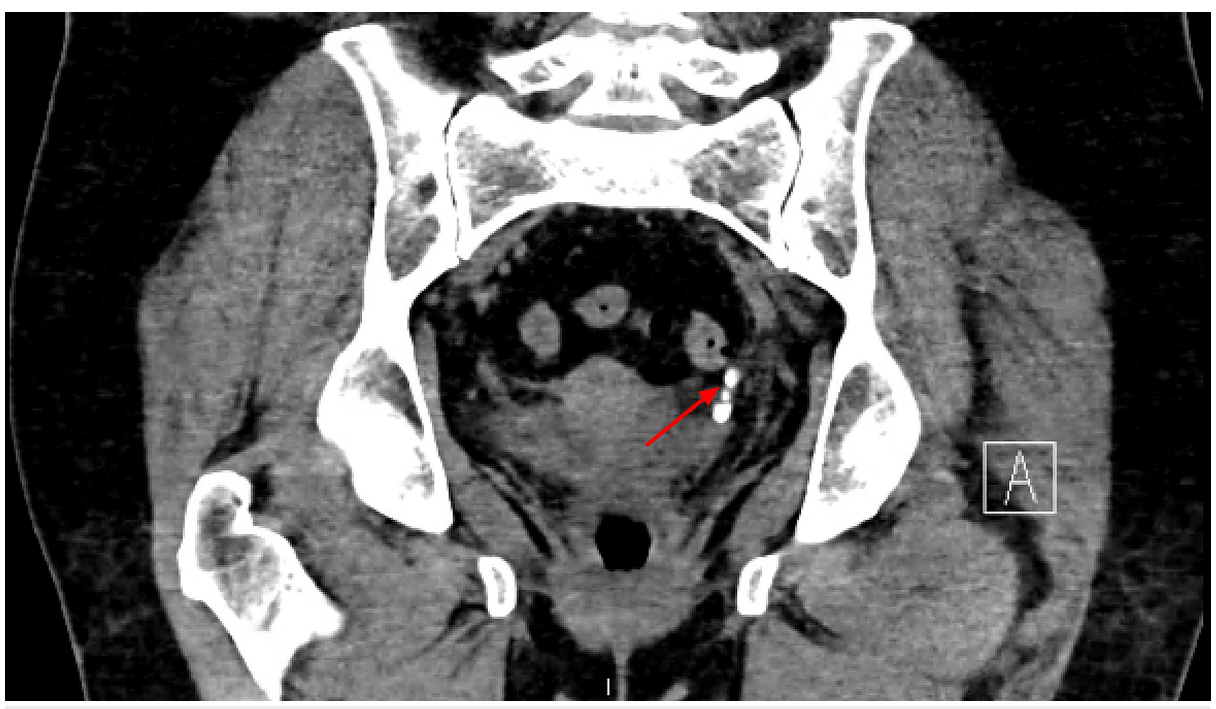

FIGURE 2: Acute obstructing calculi in the distal left ureter

Urology was consulted, and the patient underwent left ureteroscopy with a J-stent placement. Urine and blood cultures revealed Salmonella species > 100,000 colony-forming units per milliliter (CFU/mL) and NTS, respectively. The patient was treated with multiple antibiotics during her hospitalization, initially started on ceftriaxone, $1 \mathrm{~g}$ IV daily, transitioned to ampicillin, $2 \mathrm{~g}$ IV q four hrs, and then eventually to levofloxacin, 750 mg PO daily, due to persistent fever and leukocytosis. Her symptoms significantly improved on levofloxacin which was also continued on discharge for a further two weeks. On further review, it was known that patient had presented to the emergency room (ER) about four months earlier with abdominal pain and watery diarrhea with a stool culture being positive for NTS. Repeat laboratory studies, urinalysis, and urine culture at the clinic one month after hospital discharge were unremarkable with the patient being completely stable with no new symptoms.

\section{Discussion}

Gastroenteritis, sepsis, and enteric fever are normally known with S. Typhi. Less common extraintestinal 
diseases, such as urinary tract infections, are due to NTS [5]. Predisposing factors for NTS UTI include chronic diseases like diabetes, immune deficiency, or genitourinary tract abnormalities, including nephrolithiasis, chronic pyelonephritis, urethrorectal, or retrovesical fistula. It should not be ignored that NTS UTI can also occur in the healthy immunocompetent population [6].

The most frequent pathogenesis of a Salmonella upper urinary tract infection is probably hematogenous. Salmonella enters the body through ingestion and colonizes the ileum and colon. When Salmonella invades the bloodstream, it can seed distant target organs, such as the kidneys, and cause pyelonephritis. In our case, the patient had NTS gastroenteritis, followed by NTS bacteremia and UTI. Abuhasna et al. reported a similar case in which the patient had NTS UTI and was found to have blood cultures positive for NTS, making hematogenous cause a significant reason for NTS UTI [7]. Salmonella UTI has symptoms similar to other gram-negative UTIs, ranging from asymptomatic bacteriuria to renal abscess [8]. The reported duration of antibiotic therapy in a Salmonella urinary tract infection ranges from two (for mild infections) to over six weeks [9].

\section{Conclusions}

NTS infections often present with self-limiting gastroenteritis. Urinary tract infections caused by NTS are usually associated with structural abnormalities of the urinary tract and should prompt the physician to evaluate further. Nephrocalcinosis and nephrolithiasis are the major risk factors.

\section{Additional Information \\ Disclosures}

Human subjects: Consent was obtained by all participants in this study. Conflicts of interest: In compliance with the ICMJE uniform disclosure form, all authors declare the following: Payment/services info: All authors have declared that no financial support was received from any organization for the submitted work. Financial relationships: All authors have declared that they have no financial relationships at present or within the previous three years with any organizations that might have an interest in the submitted work. Other relationships: All authors have declared that there are no other relationships or activities that could appear to have influenced the submitted work.

\section{References}

1. Cohen JI, Bartlett JA, Corey GR: Extra-intestinal manifestations of salmonella infections. Medicine (Baltimore). 1987, 66:349-88. 10.1097/00005792-198709000-00003

2. Seligman E, Saphra I, Wassermann M: Salmonella infections in the U.S.A.; a second series of 2,000 human infections recorded by the N.Y. Salmonella Center. J Immunol. 1946, 54:69-87.

3. Ramos JM, Aguado JM, García-Corbeira P, Alés JM, Soriano F: Clinical spectrum of urinary tract infections due on nontyphoidal Salmonella species. Clin Infect Dis. 1996, 23:388-90. 10.1093/clinids/23.2.388

4. Batista da Costa J, Cornu JN, Levgraverend D, et al.: Pyonephrosis caused by Salmonella typhi: a case report . Urol Int. 2014, 96:241-43. 10.1159/000360741

5. Abbott SL, Portoni BA, Janda JM: Urinary tract infections associated with nontyphoidal Salmonella serogroups. J Clin Microbiol. 1999, 37:4177-78. 10.1128/JCM.37.12.4177-4178.1999

6. Jehangir A, Poudel D, Fareedy SB, Salman A, Qureshi A, Jehangir Q, Alweis R: Group D salmonella urinary tract infection in an immunocompetent male. Case Rep Infect Dis. 2015, 2015:608632. 10.1155/2015/608632

7. Abuhasna S, Al Jundi A, Rahman MU, Said W: Non-typhoidal Salmonella group D bacteremia and urosepsis in a patient diagnosed with HIV infection. J Glob Infect Dis. 2012, 4:218-19. 10.4103/0974-777X.103902

8. Paterson DL, Harrison MW, Robson JM: Clinical spectrum of urinary tract infections due to nontyphoidal Salmonella species. Clin Infect Dis. 1997, 25:754. 10.1086/513751

9. Bassily SB, Kilpatrick ME, Farid Z, Mikhail IA, El-Masry NA: Chronic Salmonella bacteriuria with intermittent bacteremia treated with low doses of amoxicillin or ampicillin. Antimicrob Agents Chemother. 1981, 20:630-33. 10.1128/aac.20.5.630 